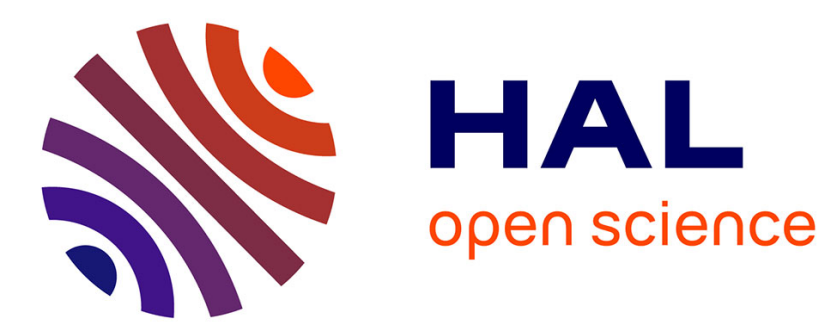

\title{
Thermal conductivity of the Lennard-Jones fluid: an empirical correlation.
}

\author{
Mathilde Bugel, Guillaume Galliero
}

\section{To cite this version:}

Mathilde Bugel, Guillaume Galliero. Thermal conductivity of the Lennard-Jones fluid: an empirical correlation.. Chemical Physics, 2008, 352 (1-3), pp.249-257. 10.1016/j.chemphys.2008.06.013 . hal00322156

\section{HAL Id: hal-00322156 https://hal.science/hal-00322156}

Submitted on 16 Sep 2008

HAL is a multi-disciplinary open access archive for the deposit and dissemination of scientific research documents, whether they are published or not. The documents may come from teaching and research institutions in France or abroad, or from public or private research centers.
L'archive ouverte pluridisciplinaire HAL, est destinée au dépôt et à la diffusion de documents scientifiques de niveau recherche, publiés ou non, émanant des établissements d'enseignement et de recherche français ou étrangers, des laboratoires publics ou privés. 


\title{
Thermal conductivity of the Lennard-Jones fluid: an empirical correlation.
}

\author{
Mathilde BUGEL ${ }^{1}$, Guillaume GALLIERO ${ }^{2,3 *}$ \\ ${ }^{1}$ Laboratoire TREFLE, UMR 8508, Université Bordeaux I, ENSCPB, 16 Avenue Pey- \\ Berland, F-33607 Pessac Cedex, FRANCE. \\ 2 Laboratoire des Fluides Complexes, UMR 5150, Université de Pau et des Pays de \\ l'Adour, Faculté des Sciences et Techniques, BP 1155, F-64013 Pau Cedex, FRANCE. \\ ${ }^{3}$ Laboratoire LETEM, Université de Marne-la-Vallée, Bât. Lavoisier, Champs-sur- \\ Marne, F-77454 Marne-la-Vallée Cedex 2, FRANCE. \\ *Corresponding author: Tel: +33 5594077 04; Fax: +33 5594076 95; email: \\ guillaume.galliero@univ-pau.fr
}

\begin{abstract}
In this work, is presented an empirical correlation on the thermal conductivity of the Lennard-Jones fluid based on extensive non-equilibrium molecular dynamics simulations results (103 points). Finite size and cutoff radius effects are investigated and taken into account to develop the correlation. This last, composed of low density, residual and critical enhancement contributions, is built for a wide range of thermodynamics states, even at the vicinity of the critical point, and yields an average absolute deviation of $1.29 \%$ compared to our simulations. In addition, a careful analysis of the different contributions to the microscopic flux is carried out which sheds light on the underlying mechanism of the results. Finally, are discussed the limitations of the proposed model when applied to real simple fluids and mixtures using a standard corresponding states scheme and the van der Waals one-fluid approximation.
\end{abstract}




\section{Introduction}

An accurate knowledge of thermal conductivity is essential in various fields such as fluid mechanics, chemical engineering, or industrial processing of materials. But, despite numerous models, an accurate modeling of this property is still lacking especially in dense fluid mixtures [1]. On way to improve the theoretical understanding of the thermal conductivity behavior in dense fluid, as well to provide a physically based correlation, is the use of molecular dynamics (MD) simulations on model fluids such as the widely used Lennard-Jones (LJ) fluid model.

Nevertheless, compared to viscosity [2-6] or even more self diffusion [7-10], there is a lack of systematic studies of the amplitude of thermal conductivity of the Lennard-Jones fluid, even if some recent results have been published [11-14]. In particular, to the best of our knowledge, it only exists one work (theoretically based) [14] which provides a relation to estimate the LJ thermal conductivity. This lack of MD data on thermal conductivity of the LJ fluid is surprising as long as it exists various efficient schemes to compute this transport property: the classical equilibrium molecular dynamics (EMD) method [15] and different non-equilibrium molecular dynamics (NEMD) approaches [13, 16-18].

So, in this work, using the simple Lennard-Jones (LJ) fluid model as a basis, a large number of NEMD simulations (based on the algorithm proposed in reference [18]) for various thermodynamic states have been performed (even close to the critical point). The goal is twofold:

-First, improve the understanding of the energy transport in LJ fluids (in particular close to the critical point) by a careful analysis of the microscopic contributions to the heat flux.

-Second, as already done by us for viscosity [2], construct a simple and accurate correlation on thermal conductivity of the Lennard-Jones fluid for a wide range of thermodynamic states. Then, quantify to which extent such a correlation, which deals only with translational mode of energy transfer, could provide a good estimation on simple real pure fluids and mixtures when combined with a standard corresponding state scheme. 
It is worth emphasizing that, apart from the two goals mentioned above, such a correlation could be useful as well when coupling computational fluid dynamics (CFD) and MD [19-23]. In fact, a very accurate knowledge of transport properties at the microscopic level of the fluid model, usually the Lennard-Jones one, is required to inject the proper quantities in the macroscopic equation, otherwise the convergence in the overlapping region will not be achieved.

In a first part, are presented the effects of the numerical parameters of the MD simulations ( $N$, number of particles, $r_{c}$, cutoff radius) on thermal conductivity. Then, based on the results of more than 100 points of simulations covering from $T^{*}=0.6$ to 4.0 and up to $\rho^{*}=0.9$, a simple correlation applicable from gas to dense systems and even close to the critical point is proposed. Simultaneously, an analysis of the results in terms of heat flux contributions is provided. In a last part, this correlation is applied to some simple pure fluids and mixtures discussing the intrinsic limitations of the LJ fluid model.

\section{Theory and model}

\subsection{Interaction potential}

To model the fluid particle interactions, we have used the Lennard-Jones 12-6 potential, which is, for a molecule $i$ interacting with a molecule $j$ :

$$
U_{i j}=4 \varepsilon_{i j}\left[\left(\frac{\sigma_{i j}}{r_{i j}}\right)^{12}-\left(\frac{\sigma_{i j}}{r_{i j}}\right)^{6}\right]
$$

where $\varepsilon_{i j}$ is the potential strength, $\sigma_{i j}$ the distance at which the potential is equal to zero, and $r_{i j}$ the intermolecular distance.

When dealing with mixtures, the crossmolecular parameters between unlike particles $i$ and $j$ appearing in eq. (1) have to be defined using a set of combining rules. In this work, we have chosen to use the classical Lorentz-Berthelot (LB) combining rules:

$$
\begin{gathered}
\sigma_{i j}=\left(\frac{\sigma_{i i}+\sigma_{j j}}{2}\right) \\
\varepsilon_{i j}=\left(\varepsilon_{i i} \varepsilon_{j j}\right)^{1 / 2}
\end{gathered}
$$




\subsection{Microscopic law of the corresponding states}

The law of the corresponding states postulates that, with an adequate scaling procedure, different fluids, at the same reduced conditions, have superposing thermodynamic phase diagrams. In this scheme, the reduced LJ temperature and density are respectively defined by:

$$
\begin{aligned}
& T^{*}=\frac{k_{B} T}{\varepsilon_{x}} \\
& \rho^{*}=\frac{N \sigma_{x}^{3}}{V}
\end{aligned}
$$

where $k_{B}$ is the Boltzmann constant, $T$ the temperature, $N$ the number of particles, $V$ the volume of the simulation box, $\sigma_{x}$ and $\varepsilon_{x}$ the characteristic LJ potential parameters of the studied fluid. The reduced pressure which is a unique function of $T^{*}$ and $\rho^{*}$ for the LJ potential is:

$$
P^{*}=\frac{P \sigma_{x}^{3}}{\varepsilon_{x}}
$$

where $P$ is the pressure of the system.

In addition, this microscopic formulation of the corresponding states implies that, for a given potential shape, reduced dynamic properties are unique function of $T^{*}$ and $\rho^{*}$; for the thermal conductivity such an approach yields:

$$
\lambda^{*}=\lambda \frac{\sigma_{x}^{2}}{k_{B}}\left(\frac{m_{x}}{\varepsilon_{x}}\right)^{1 / 2}
$$

where $m_{x}$ is the characteristic molecular mass of the fluid and $\lambda$ the thermal conductivity.

\section{3. van der Waals one-fluid approximation}

In mixtures, $\sigma_{x}, \varepsilon_{x}$ and $m_{x}$, appearing in eqs. (4-7), have to be defined by a one-fluid approximation which allows to describe the mixture as a single pseudocomponent "equivalent" to the mixture. To do so, we have chosen the van der Waals one-fluid approximation (vdW1) which provides usually reasonable results:

$$
m_{x}=\sum_{i} x_{i} m_{i}
$$




$$
\begin{gathered}
\sigma_{x}^{3}=\sum_{i} \sum_{j} x_{i} x_{j} \sigma_{i j}^{3} \\
\varepsilon_{x} \sigma_{x}^{3}=\sum_{i} \sum_{j} x_{i} x_{j} \varepsilon_{i j} \sigma_{i j}^{3}
\end{gathered}
$$

where the $x_{i}$ are the molar fractions and $\sigma_{i j}$ and $\varepsilon_{i j}$ are calculated using eqs. (2) and (3).

\subsection{Heat flux decomposition}

During the stationary state of the non equilibrium molecular dynamics simulations, it is possible to estimate the local instantaneous internal energy flux, $\mathbf{J}_{\mathrm{U}}$, by using its microscopic formulation (Irving-Kirkwood) which is composed of three terms [18]:

$$
J_{U}=J_{k}+J_{p}+J_{c o}
$$

where $\mathbf{J}_{\mathrm{k}}$ corresponds to the kinetic energy transferred by a moving particle, given by:

$$
\mathrm{J}_{\mathrm{k}}=\frac{1}{V_{C}} \sum_{i}\left(\frac{1}{2} m_{i}\left(\mathrm{v}_{i}-\mathrm{v}\right)^{2}\right)\left(\mathrm{v}_{i}-\mathrm{v}\right)
$$

$\mathbf{J}_{\mathrm{p}}$ corresponds to the potential energy transferred by a moving particle, given by:

$$
\mathrm{J}_{\mathrm{p}}=\frac{1}{V_{C}} \sum_{i} \phi_{i}\left(\mathrm{v}_{i}-\mathrm{v}\right)
$$

and $\mathbf{J}_{\mathrm{co}}$ is the energy transferred by collision with other particles:

$$
\mathrm{J}_{c o}=-\frac{1}{V_{C}} \sum_{i} \sum_{j>i}\left[\left(\mathrm{v}_{i}-\mathrm{v}\right) \cdot \mathrm{F}_{i j}\right] \mathrm{r}_{i j}
$$

where $V_{c}$ is the control volume, $\mathbf{v}_{\mathrm{i}}$ the velocity of particle $i, \mathbf{v}$ the barycentric velocity of the system, $\phi_{i}$ the potential energy of particle $i$ in the field of all other particles, $\mathbf{F}_{i j}$ the force acting on $i$ due to $j$ and $\mathbf{r}_{i j}$ is the vector from the position $i$ to the position $j$.

In addition, in pure fluids, the internal energy flux, $\mathbf{J}_{\mathrm{U}}$, computed by eq. (11) is equal to the heat flux, $\mathbf{J}_{\mathrm{q}}$, given by the Fourier's law:

$$
\mathbf{J}_{q}=-\lambda \nabla T
$$

which is not the case in mixtures if the mass fluxes are not equal to zero [24].

Thus, in the linear regime, it is possible to consider that the thermal conductivity is composed of three terms:

$$
\lambda=\lambda_{K}+\lambda_{p}+\lambda_{c o}
$$

where $\lambda_{k}$ and $\lambda_{p}$ correspond respectively to the kinetic and potential contributions and $\lambda_{c o}$ to the collisionnal part (configurational). The two first quantities could be gathered 
to form the translational contribution. It is worth noticing that such a decomposition is different from the one sometimes used in equilibrium molecular dynamics simulations [11], which induces byproducts between translational and configurational contributions.

\subsection{Non-Equilibrium Molecular Dynamics}

One major purpose of this study is to have a reliable database on the reduced thermal conductivity $\lambda^{*}$ of the LJ fluids. To compute this transport property in Lennard-Jones fluids using molecular dynamics, a large number of techniques are available. They are based on either equilibrium [15], synthetic nonequilibrium [17], or boundary driven non-equilibrium approaches $[13,16,18]$. Among them we have chosen a boundary nonequilibrium molecular dynamics scheme, the HEX (Heat EXchange) algorithm developed by Hafskjold et al. [18], simple to handle and providing reliable results. In this scheme a biperiodical heat flux is imposed to the simulation, and after a transient state, the thermal conductivity is deduced using the Fourier's law and the measured temperature profile.

To do so, the simulation box is divided into $N_{\mathrm{s}}$ slabs (in our simulations $N_{\mathrm{s}}=32$ ), of identical thickness and volume, along the $\mathrm{z}$ direction, see figure 1. Slabs 1 and $N_{s}$ are defined as the "hot" ones and slabs $N_{s} / 2$ and $N_{s} / 2+1$ as the "cool" ones. The heat flux is generated by an exchange, at each time step, of kinetic energy between the cool slabs, at the center, and the hot slabs, at the extremities of the box, so that the temperature increases in the hot slabs and decreases in the cool slabs (while keeping the linear momentum constant). By conduction, the generated heat flux,

$$
\mathbf{J}_{q}^{i m p}=\frac{\Delta E_{c}}{2 t L_{x} L_{y}}
$$

where $\Delta \mathrm{E}_{\mathrm{c}}$ is the kinetic energy exchanged during the simulation time $t ; L_{x}, L_{y}$, the box lengths in $x$ and $y$ directions, leads to a temperature gradient. The slabs where the exchanges are performed, as well as their first neighbors, have been discarded to measure the temperature gradient because of non linear behavior in these regions [18], see figure 2 .

It should be noted that, in mixtures, the thermal conductivity deduced using this scheme corresponds to an effective thermal conductivity, which is composed by part of a thermodiffusion (Dufour effect) contribution [24] due to the fact that a molar fraction 
gradient appears. Nevertheless, in systems studied here this contribution is at the highest a few percent only.

Besides, as long as the Irving-Kirkwood microscopic formulation of the heat flux, eqs. (11)-(14), is strictly speaking valid only in homogeneous systems [25] (which is not the case in the NEMD scheme chosen) we have verified that the ratio between the imposed heat flux, eq. (17) and the computed one, using eq. (11), is always close to unity. It has been found that the deviations were lower than $1 \%$ which means that the procedure is consistent.

\subsection{Technical details}

The code used is homemade [26] and uses the Verlet velocity algorithm [15] to integrate the equations of motion. The Verlet neighbor list coupled with a cell list method [15] has been used to save CPU time. The reduced time step, $\delta t^{*}=\delta t \frac{\sqrt{\varepsilon_{x} / m_{x}}}{\sigma_{x}}$, has been taken equal to 0.002. Usual periodic boundary conditions and minimum image convention were applied. To avoid a temperature drift during the simulation, we have used a Berendsen thermostat with a very long coupling constant to perturb as less as possible the system, this constant being equal to 1000 time steps [27]. It has been verified that such thermostat does not affect the heat flux contribution (see section 2.4) results. Simulations have been performed on systems composed of 1500 Lennard-Jones 12-6 spheres, having a $2.5 \sigma$ cutoff radius, except when stated. The influence of these numerical parameters is discussed in section 3 .

After equilibration and the NEMD transient state (at least $10^{5}$ time steps) simulations were performed during at least $2.5 .10^{6}$ nonequilibrium time steps (five runs of $5.10^{5}$ time steps each). To avoid a non-linear response of the system, a relatively weak reduced heat flux, $\mathbf{J}_{q}^{*}=\mathbf{J}_{q} \frac{\sigma_{x}}{\varepsilon_{x}} \sqrt{\frac{m_{x}}{\varepsilon_{x}}}$, ranging from 0.07 to 0.3 , depending on the thermodynamic state, has been applied. It should be noted that, for each simulation, a great care has been taken to obtain a linear thermal gradient and to avoid phase transition (by analyzing the density gradient). An example of one temperature profile is addressed in figure 2. Using these parameters, the estimated errors (using the subblocks 
average method [15] with subblock composed of $5.10^{5}$ timesteps to ensure the statistical independence) lie between 1 and $9 \%$, depending on the state, see the appendix.

\section{Preliminary results}

\subsection{System size}

By performing simulations on systems composed of 500 to 8000 particles, we have studied the influence of the system size on the thermal conductivity computed by the NEMD scheme we used [18], for four thermodynamics states $\left(\rho^{*}=0.3\right.$ and $0.8, T^{*}=1$ and 2.5). As the simulation box is always cubic, width, height and depth vary in the same proportion.

Contrary to what has been found recently by Mountain [28], which uses a similar nonequilibrium algorithm (but keep $L_{z}$ constant when varying $N$ ), non-negligible finite size effects have been noticed, see figure 3, as noted previously in a supercritical state [26]. In addition, it has been found that these finite size effects are dependent on the thermodynamic states as noted recently by Heyes et al. [29] for the self diffusion of the hard-sphere fluids, see figure 3. The underestimation of thermal conductivity observed increases with $T^{*}$ and decreases with $\rho^{*}$.

Quite interestingly, as it is shown in figure 3, we found a linear decrease of $\lambda^{*}$ with $N^{1 / 2}$ whatever the thermodynamic state. It should be noted, as shown in [29], that due to the system size accessible, the discrimination between a dependence with $N^{1 / 2}$ or the more usual $N^{1 / 3}$ is hard to achieve. For the case studied here, the $N^{1 / 2}$ scaling provides slightly better results than the $N^{-1 / 3}$ one. Using this finding, we propose a corrective term that enables to deduce values of the thermal conductivity in the thermodynamic limit $\lambda_{\infty}^{*}$, from the values obtained for a finite number of particle, $\lambda^{*}$ :

$$
\lambda_{\infty}^{*}=\lambda^{*}\left(1+\alpha N^{-0.5}\right)
$$

where $\alpha$ is a function of $T^{*}$ and $\rho^{*}$.

To estimate $\alpha$, following the trends noticed in figure 3 , we have assumed that this correction could be simply expressed as:

$$
\alpha=A T^{*}+\frac{B}{\rho^{*}}
$$


where $A$ and $B$ are numerical constants. By regressing these coefficients on the simulations results we have obtained $A=0.7436$ and $B=1.1193$, results are shown in figure 3.

By analyzing the dependence of the three contributions to the thermal conductivity, see eq. (16), further information could be gathered. First, see figure 4 , it appears that all three contributions are affected by finite size effects, and all scale as $N^{1 / 2}$. In addition, the two translational contributions, $\lambda_{k}{ }^{*}$ and $\lambda_{p}{ }^{*}$, are approximately two times more affected by the size of the system than the collisional contribution, $\lambda_{c o}{ }^{*}$. This means that the behavior of $\lambda^{*}$ with the size of the system is mainly related to the one of the translational contribution $\left(\lambda_{k}{ }^{*}+\lambda_{p}{ }^{*}\right)$. Such a result explains why the dependence of $\lambda^{*}$ on the size of the system increases with $T^{*}$ and decreases with $\rho^{*}$ because an increase of temperature and/or a decrease of density favors the transfer of energy by a moving particle (translational contribution) relatively to the transfer of energy by collisions (collisional contribution). This point is dealt with in details in section 4.4.

\subsection{Cutoff radius}

Cutoff radius may affect the amplitude of the computed transport properties, as for instance when dealing with thermodiffusion [26]. Therefore, simulations for various cutoff radius (between 2 and $4 \sigma$ ) have been performed for four different thermodynamics states, see figure 5, on a fixed number of particles, equal to 1500 .

For the tested thermodynamic states, it has been found that the thermal conductivity values do not appear to be sensitive to the cutoff radius value. More precisely, values obtained for different $r_{c}$ lie within the error bars of each other, see figure 5 , as noticed previously in a supercritical state [30] and for other direct transport properties in the liquid state by Meier [11]. This trend is consistent with the fact that direct transport properties (like viscosity, mass diffusion or thermal conductivity) are believed to primarily depend on the occurrence of collisions and not on the nature of the collisions (related to the shape of the potential). Therefore, in the following, we have used the $2.5 \sigma$ cutoff radius $\left(r_{c}{ }^{*}=2.5\right)$ which appears to be a good compromise between reliable results and CPU time needs for the computation of thermal conductivity and which is probably the most widely used in the literature. 


\subsection{Validation of results with regard to literature}

As a test concerning the values provided by our simulations (taking into account the finite size correction, eqs. (18-19)), a comparison with the recent data obtained by Nasrabad et al. [14] (which uses an EMD approach) and Mountain [28] (which compares NEMD and EMD results) has been performed. Thermodynamic conditions simulated cover from $T^{*}=0.7$ to $T^{*}=3.6$ and from $\rho^{*}=0.3$ to $\rho^{*}=0.9$. To estimate the reliability of our results, we have calculated the bias, the average absolute deviation (AAD) and the maximal deviation $\left(\Delta_{\max }\right)$ between our values and those coming from references [14] and [28]:

$$
\begin{gathered}
\text { bias }=\frac{1}{N_{c}} \sum_{i=1}^{N_{c}} 100\left(1-\frac{\lambda_{\text {thiswork }}^{*}}{\lambda_{\text {Nas./Moun. }}^{*}}\right) \\
A A D=\frac{1}{N_{c}} \sum_{i=1}^{N_{c}} 100\left|1-\frac{\lambda_{\text {thiswork }}^{*}}{\lambda_{\text {Nas./Moun. }}^{*}}\right| \\
\Delta_{\max }=\max \left(100\left|1-\frac{\lambda_{\text {thiswork }}^{*}}{\lambda_{\text {Nas./Moun. }}^{*}}\right|\right)
\end{gathered}
$$

All these quantities are expressed in percentages.

The results are summarized in Table 1 and they clearly indicate that our values are consistent with recent literature data (using both EMD and NEMD approaches), even if our values, on average, slightly overestimate those of references [14] and [28] (around 3 $\%)$. This slight discrepancy may probably be imputed to the fact that no finite size effects have been taken into account in their simulations.

\section{Thermal conductivity correlation}

\subsection{Reduced thermal conductivity expression}

As proposed usually in the literature [31-32], to construct the thermal conductivity correlation, we have assumed that $\lambda^{*}$ can be expressed as a sum of a low-density 
contribution, $\lambda_{0}{ }^{*}$, a residual thermal conductivity $\lambda_{r}{ }^{*}$, and a critical thermal conductivity enhancement $\lambda_{c}^{*}$ :

$$
\lambda^{*}\left(T^{*}, \rho^{*}\right)=\lambda_{0}^{*}\left(T^{*}\right)+\lambda_{r}^{*}\left(T^{*}, \rho^{*}\right)+\lambda_{c}^{*}\left(T^{*}, \rho^{*}\right)
$$

For $\lambda_{0}{ }^{*}$, we use the first-order approximation of the Chapman-Enskog approach applied to the $\mathrm{LJ}$ potential which, in reduced units, is:

$$
\lambda_{0}^{*}\left(T^{*}\right)=\frac{75}{64 \Omega_{v}}\left(\frac{T^{*}}{\pi}\right)^{1 / 2}
$$

where $\Omega_{v}$ is the collision integral, which is a function of $T^{*}$, that can be estimated using the correlation proposed by Neufeld et al. [33], applicable for $T^{*}$ ranging from 0.3 to 100.

To model the residual thermal conductivity, resulting from the interaction between particles, a simple heuristic formulation, inspired by the work of Nasrabad et al. [14] and the one of Lemmon et al. [32], is proposed. The dependence of $\lambda_{r}{ }^{*}$ with density and temperature is modeled by:

$$
\lambda_{r}^{*}\left(\rho^{*}, T^{*}\right)=A\left(T^{*}\right)\left[\exp \left(B\left(T^{*}\right) \rho^{* \frac{2}{3}}\right)-1\right]
$$

where $A$ and $B$ are linear functions of the reduced temperature:

$$
\begin{aligned}
& A\left(T^{*}\right)=C T^{*}+D \\
& B\left(T^{*}\right)=E T^{*}+F
\end{aligned}
$$

where $C, D, E, F$ are coefficients fitted on molecular dynamics simulations results.

Lastly, $\lambda_{c}{ }^{*}$, which characterizes the enhancement due to the proximity of the critical point, is given by the following expression which relates this enhancement to the isothermal compressibility one as it is done in the work of Mathias et al. [34]:

$$
\lambda_{c}^{*}=a\left(T^{*} \rho^{* 2} \chi_{T}^{*}\right)^{b}
$$

where $\chi_{T}{ }^{*}$ is the reduced isothermal compressibility and $a, b$ two positive coefficients fitted on molecular dynamics results. To calculate $\chi_{T}{ }^{*}$ we have used the LJ fluid equation of state (EOS) of Kofala and Nezbeda [35]. Besides, it is worth emphasizing that such a formulation, contrary to the one proposed by Matthias et al. [34], implies that $\lambda_{c}{ }^{*} \rightarrow 0$ when $\rho^{*} \rightarrow 0$ (and so $\lambda^{*} \rightarrow \lambda_{0}{ }^{*}$ when $\rho^{*} \rightarrow 0$ as expected). 


\subsection{Critical enhancement}

As mentioned in the previous section, it is well known that close to the critical point, the thermal conductivity tends to diverge following a scaling law [36]. Contrary to viscosity, this enhancement is not restricted to the immediate vicinity of the critical point and is noticeable using molecular dynamics simulations, even if such results are scarce $[11,37]$. Nevertheless, in molecular simulations, this enhancement due to long range correlations could not properly be caught because of the finite size of the system simulated. In addition, the way this contribution is modeled in this work, eq. (28), implies the computation of $\chi_{T}$ through an equation of state, the one of Kolafa and Nezbeda [35], which is not particularly aimed at taking into account the effects of the critical point. Therefore, the way the critical enhancement is modeled in this work could only be considered as a first attempt.

To adjust parameters $a$ and $b$ of eq. (28), we have performed simulations on the isotherm $T^{*}=1.35$ (close to the critical one), for 11 values of $\rho^{*}$ varying from 0.25 to 0.4 (values that are believed to embody the critical one). Large systems composed of 8000 particles have been used. Simulations have been performed during at least $5.10^{6}$ time steps. A particularly weak reduced heat flux equal to 0.0025 has been used. In addition, to augment the fitting database, EMD values coming from Meier [11] have been added for the same isotherm (with two sets of values for $\rho^{*}=0.3$ and 0.35 ). It should be noted that the difference between EMD (Meier) and NEMD (ours) values is inherent to the NEMD method. Even if we apply a particularly small reduced heat flux, a weak temperature gradient is induced, so the simulations are not performed exactly on the isotherm $T^{*}=1.35$, contrary to those of Meier.

Figure 6 clearly shows a thermal conductivity enhancement, for both types of simulations, with a maximum (up to $25 \%$ of the total value) located close to $\rho^{*}=0.3$ which is consistent with the location of the critical point (which is located close to $\rho_{c}{ }^{*}$ $=0.3108$ and $T_{c}{ }^{*}=1.3396$ [35]). In addition, it appears that the model chosen to describe $\lambda_{\mathrm{c}}{ }^{*}$ is able to correctly reproduce the MD results when using eq. (28) with $a=0.11$ and $b=0.45$, see figure 6 . 


\subsection{Correlation regression of the residual contribution}

To regress the four coefficients needed in our formulation of the residual contribution, $\lambda_{\mathrm{r}}{ }^{*}$, we have used a database composed of 92 new points of simulations of our own, in addition to the 11 points on the isotherm $T^{*}=1.35$ (see previous section), covering from $0.3 \leq \rho^{*} \leq 0.9$ and $0.6 \leq T^{*} \leq 4$. The thermal conductivity values used for the regression take into account the finite size correction, eq. (18) and eq. (19) and are provided in the appendix. The regression was performed in order to minimize $\Delta$ max, with a bias constrained to zero. The coefficients obtained are summarized in Table 2.

The AAD between the MD values and those obtained with the correlation is equal to $1.29 \%$, and the $\Delta \max$ is equal to $4.12 \%$. All deviations are shown in figure 7 . If the points located on the close critical isotherm $T^{*}=1.35$ are omitted then AAD $=1.18 \%$ and $\Delta \max =3.22 \%$. Compared to the inherent statistical errors of the MD simulations, and to the wide range of thermodynamic conditions tested, even close to the critical point, such a low AAD can be considered as excellent. Thus, this simple empirical correlation adequately represents the thermal conductivity of our NEMD results on the LJ fluid over the domain $0 \leq \rho^{*} \leq 0.9$ and $0.6 \leq T^{*} \leq 4$, which covers gas, liquid, and supercritical phases even in the vicinity of the critical point.

As a further test of the correlation proposed, see figures 7 and 8, we have applied it on data coming from the literature which uses different approaches to compute thermal conductivity, EMD for the work of Nasrabad et al. [14] and a new NEMD scheme for the work of Hulse et al. [13]. In addition, the EMD and NEMD results of Mountain [28] have been added for the comparison. These figures clearly exhibit that there is a good agreement between the results provided by our correlation and those coming from the literature, the deviations being always lower than $14 \%$. Besides, it is worth noticing that our correlation yields results closer to the EMD data of Nasrabad et al. [14] than their own theory. Our correlation yields $\mathrm{AAD}=3.74 \%$ with $\Delta_{\max }=8.28 \%$, whereas their model yields $\mathrm{AAD}=14.92 \%$ and $\Delta_{\max }=78.62 \%$.

\subsection{Heat flux contributions}

On figure 8 are shown the relative contributions to the heat flux for three different isotherms, $T^{*}=1,1.35$ and 2.5 , versus the reduced density. As expected, the collisional contribution monotonically increases with density (in a non-linear way) and decreases 
with temperature whereas the kinetic one decreases with density and increases with temperature in a symmetric manner. For the range of temperatures tested, these two contributions become of the same order for $\rho^{*} \approx 0.3-0.4$.

The case of the potential contribution is more interesting, see figure 8 . This contribution is always rather small, around $5 \%$ of the whole heat flux as already noticed by [38], and weakly depends on the density, except close to the critical point where its contribution climbs up to $14 \%$. Thus, we can suspect that the enhancement of the thermal conductivity (close to the critical point) is related to the increase of the potential heat flux contribution. This finding is consistent with the abnormal behavior of thermal conductivity in nanofluids (unexpected increase compared to value in the pure fluid), which seems to exhibit the same peculiar increase of the potential contribution to the heat flux [38].

\section{Numerical results for real fluids}

The Lennard-Jones fluid model has shown to be able to provide reasonable results concerning the prediction of viscosity and mass diffusion of simple fluids for a large variety of thermodynamic states, see for instance $[2,3,7-8]$. So, we have applied our correlation for the prediction of the thermal conductivity of real fluids to see to which extent such an approach is applicable.

In order to use a unique set of molecular parameters to predict all thermophysical properties, we have taken the values of the molecular parameters, $\varepsilon$ and $\sigma$, from [39]. These parameters have been adjusted in order to reproduce both pressure and viscosity and are provided in Table 3 for the species studied here. Then, using the correlation, eqs. (23)-(28), and the relations (4)-(7), it is possible to obtain the predicted thermal conductivity for a peculiar species in the chosen state $(T, \rho)$. Additionally, for mixtures, the Lorentz-Berthelot combining rules, eqs. (2) and (3) are used to define the crossmolecular parameters, and eqs. (8)-(10) are applied for the calculation of the pseudocomponent representative of the mixture (vdW1). It should be emphasized that the scheme proposed here to predict thermal conductivity is entirely predictive 


\subsection{Pure compounds}

We have first applied the correlation to four simple nonpolar molecules, Argon, Methane, Oxygen and Nitrogen for a large range of thermodynamic states (which cover gas, liquid and supercritical states), see Table 4. The database used for comparison is based on specific correlations for each compound, which corresponds to the best possible fit of a large variety of experimental results [40-43].

Results shown in Table 4 clearly indicate that the scheme proposed is able to provide a good estimation of Argon thermal conductivity, with a slight systematic overestimation see figure 9, but yields non negligible deviations (underestimation) for non monoatomic molecules. More precisely, except for nitrogen, predictions are reasonable in dense phase (deviations being $<10 \%$ ) and increase in more diluted ones (deviations varying from 15 to $55 \%$ ), as it can be seen in figure 9. Thus, as expected, the scheme seems to be acceptable only for strictly spherical molecules like noble gases and provides generally better results in dense phases for non spherical molecules.

In fact, the LJ fluid suffers from various weaknesses. First, it represents the decay of the repulsive interaction between particles by an inverse twelve-power dependence on intermolecular separation, which was chosen mainly for mathematical convenience and has no physical soundness [44]. Second, the molecular parameters have not been readjusted on thermal conductivity values, see the preceding section, which explain as well why results on Argon are not as good as those on viscosity [39]. Third, and even more important for thermal conductivity in Methane, Oxygen and Nitrogen, this model relies on a spherical approximation of the molecule without intramolecular interactions. Therefore, neither rotational nor vibrational degrees of freedom modes are taken into account [1]. So, the nonsphericity of the molecule as well as low density and high temperature (which favors rotation and vibration) will increase the underestimation of the proposed approach compared to experiments as it has been noticed, see figure 9. Thus, a possible first outlook of this study would consist to extend the proposed thermal conductivity formulation (valid only for translation modes) to take into account rotation modes through a third molecular parameter related to the non sphericity (by doing a similar work on Lennard-Jones chains as done for mass diffusion in reference [45] for example). 


\subsection{Case of mixtures}

We have then applied our correlation on two simple liquid binary mixtures $(\mathrm{Ar} / \mathrm{Kr}$ and $\left.\mathrm{Ar} / \mathrm{CH}_{4}\right)$ for which data were available in the work of Mikhailenko et al. [46-47]. For both systems, $x_{\mathrm{Ar}}$ varies from 0 to 1 with a step of 0.2 . Thermodynamic conditions are provided in Table 5.

Results in Table 5 show apparently that the proposed correlation combined with the vdW1 is rather efficient to predict the thermal conductivity in mixtures compared to what it produces on pure fluids (where non negligible deviations may occur, see preceding section), see Table 4. Nevertheless, contrary to what was expected, we have obtained very satisfying results on the $\mathrm{Ar}-\mathrm{CH}_{4}$ system whereas in $\mathrm{Ar}-\mathrm{Kr}$ mixtures results are only reasonable. In fact, it can be deduced from figure 10, for the $\mathrm{Ar}-\mathrm{CH}_{4}$ mixture, that there is a balance between the overestimation of thermal conductivity for pure argon and the underestimation of $\lambda$ for pure methane, which explains the abnormally good results obtained, see Table 5 .

Concerning the Ar-Kr mixture, the deviations yielded by the correlation are larger in mixtures than on pure fluids (with a maximum located around $x_{\mathrm{Ar}} \approx 0.7$ ), see figure 10 . This could be due to a weakness of the vdW1 which has been already noticed for viscosity prediction in asymmetric mixtures [48-51]. To verify this hypothesis, we have performed one simulation on the Ar-Kr mixture for the case where the deviation is the highest, i.e $x_{\mathrm{Ar}}=0.6$ at $T=140 \mathrm{~K}(22.4 \%$ of overestimation when using the vdW1). We have found that the result of the simulation on the mixture is improved compared to the one provided by the vdW1 but still overestimates the experimental value by $15 \%$, this deviation being more important than the one on pure fluids. Therefore, we can deduce that the overestimation of the thermal conductivity comes not only from the one fluid approximation, but as well from another reason which may be the Dufour effect (see section 2.5), the combining rules used or that experimental results of Mikhailenko et al. [46] are partly incorrect (they noticed a non monotonic behavior of $\lambda$ with $x_{\mathrm{Ar}}$ which is rather surprising).

\section{Conclusions and outlooks}

In this work, an accurate empirical correlation for the thermal conductivity of the LJ fluid, using NEMD simulations results, has been built. In a first part, it is shown that the 
cutoff radius does not affect the simulations results on thermal conductivity whereas finite size effects, increasing with $T^{*}$ and decreasing with $\rho^{*}$, which scale as $N^{-1 / 2}$, are noticed. These trends are explained by looking carefully to the three contributions to the microscopic heat flux (potential, kinetic and collisional). It appears that all three contributions are affected by finite size effects (and all scale as $N^{-1 / 2}$ ) but the two translational ones (potential + kinetic), predominant over the collisional one when increasing $T^{*}$ and decreasing $\rho^{*}$, are the most affected. Using the simulations results, a simple empirical finite size effects correction has been proposed.

Then, simulations have been performed on an isotherm close to the critical one $\left(T^{*}=1.35\right)$, and the results show unambiguously a clear enhancement of the thermal conductivity (up to $25 \%$ of the total value) close to the critical density $\left(\rho^{*} \approx 0.3\right)$. In addition, it has been noticed that this enhancement corresponds to an abnormal increase of the potential contribution to the heat flux ( $15 \%$ of the total heat flux) compared to what occurs in sub and supercritical states (usually below 5\% of the total heat flux).

Taking into account the correction to finite size effects and the critical enhancement, a simple heuristic correlation on thermal conductivity based on 103 new points of NEMD simulations has been constructed. This correlation is composed of low-density (using a classical Chapman-Enskog approach), residual and critical enhancement contributions, this last being related to isothermal compressibility. The proposed correlation has been adjusted in order to be efficient for $0 \leq \rho^{*} \leq 0.9$ and $0.6 \leq T^{*} \leq 4$ (which covers gas, liquid, supercritical phases), even the vicinity of the critical point. The average absolute deviation between simulations and the correlation is $1.29 \%$ and the maximum deviation is $4.12 \%$, which can be considered as excellent, compared to the large variety of states covered. In addition, it has been shown that this correlation compares favorably with literature data on the LJ thermal conductivity and is by far more accurate than the theoretical scheme proposed by Nasrabad et al [14].

In a last part, the correlation, combined with a standard corresponding states scheme, has been applied to simple real fluids, pure compounds and mixtures, for a large range of thermodynamic states. Using molecular parameters adjusted on pressure and viscosity, this entirely predictive scheme is shown to provide reasonable results on pure simple fluids ( $\mathrm{Ar}, \mathrm{N}_{2}, \mathrm{O}_{2}, \mathrm{CH}_{4}$ ) especially in dense phases. However, peculiarly for low $\rho^{*}$ and high $T^{*}$, the scheme proposed shows its limitations (underestimation) for 
polyatomic molecules which store energy in rotational and vibrational modes which are not taken into account in the proposed scheme. Finally, results for two simple liquid mixtures (Ar- $\mathrm{Kr}$ and $\mathrm{Ar}-\mathrm{CH}_{4}$ ) have been deduced thanks to a van der Waals one-fluid approximation combined with Lorentz-Berthelot rules. It is shown that this scheme provides good results on such systems, the deviations being of the order of those in pure fluids. Nevertheless, compared to experimental results, unexpected deviations were noticed for the Ar-Kr mixture (up to $22 \%$ ) that could not be imputed to the one fluid approximation alone.

\section{Acknowledgment}

This work is a part of the DSC project funded by ESA and TOTAL. We thank the TREFLE laboratory in Bordeaux and the CINES in Montpellier for having provided a large part of the computer time needed for this work. B. Duguay and J.-P. Caltagirone are acknowledge for fruitful discussions. 


\section{Appendix}

Thermal conductivity of the LJ fluid.

\begin{tabular}{|c|c|c|c|c|c|c|c|c|}
\hline $\bar{T}^{*}$ & $\rho$ & $\lambda$ & $T^{*}$ & $\rho$ & $\lambda$ & $T^{*}$ & $\rho$ & $\lambda$ \\
\hline 0.600 & 0.750 & $5.239 \pm 0.166$ & 1.300 & 0.650 & $4.092 \pm 0.151$ & 1.800 & 0.600 & $3.838 \pm 0.174$ \\
\hline 0.600 & 0.800 & $5.885 \pm 0.156$ & 1.300 & 0.700 & $4.872 \pm 0.173$ & 1.800 & 0.650 & $4.482 \pm 0.195$ \\
\hline 0.900 & 0.650 & $3.914 \pm 0.101$ & 1.300 & 0.750 & $5.813 \pm 0.210$ & 1.800 & 0.700 & $5.303 \pm 0.276$ \\
\hline 0.900 & 0.700 & $4.549 \pm 0.109$ & 1.300 & 0.800 & $6.844 \pm 0.292$ & 1.800 & 0.750 & $6.144 \pm 0.315$ \\
\hline 0.900 & 0.750 & $5.398 \pm 0.130$ & 1.300 & 0.850 & $7.966 \pm 0.395$ & 1.800 & 0.800 & $7.316 \pm 0.420$ \\
\hline 0.900 & 0.800 & $6.363 \pm 0.181$ & 1.300 & 0.900 & $9.330 \pm 0.472$ & 1.800 & 0.850 & $8.481 \pm 0.519$ \\
\hline 0.900 & 0.850 & $7.471 \pm 0.247$ & 1.350 & 0.250 & $1.337 \pm 0.102$ & 1.800 & 0.900 & $9.853 \pm 0.613$ \\
\hline 0.900 & 0.900 & $8.617 \pm 0.285$ & 1.350 & 0.275 & $1.464 \pm 0.091$ & 2.000 & 0.300 & $1.699 \pm 0.059$ \\
\hline 1.000 & 0.600 & $3.403 \pm 0.083$ & 1.350 & 0.288 & $1.546 \pm 0.123$ & 2.000 & 0.400 & $2.171 \pm 0.089$ \\
\hline 1.000 & 0.650 & $3.932 \pm 0.110$ & 1.350 & 0.300 & $1.652 \pm 0.087$ & 2.000 & 0.450 & $2.498 \pm 0.114$ \\
\hline 1.000 & 0.700 & $4.627 \pm 0.123$ & 1.350 & 0.306 & $1.677 \pm 0.132$ & 2.000 & 0.500 & $2.916 \pm 0.140$ \\
\hline 1.000 & 0.750 & $5.497 \pm 0.149$ & 1.350 & 0.313 & $1.747 \pm 0.145$ & 2.000 & 0.550 & $3.448 \pm 0.169$ \\
\hline 1.000 & 0.800 & $6.499 \pm 0.208$ & 1.350 & 0.319 & $1.706 \pm 0.117$ & 2.000 & 0.600 & $3.962 \pm 0.197$ \\
\hline 1.000 & 0.850 & $7.575 \pm 0.280$ & 1.350 & 0.325 & $1.700 \pm 0.109$ & 2.000 & 0.650 & $4.653 \pm 0.233$ \\
\hline 1.000 & 0.900 & $8.807 \pm 0.329$ & 1.350 & 0.338 & $1.755 \pm 0.099$ & 2.000 & 0.700 & $5.412 \pm 0.306$ \\
\hline 1.100 & 0.600 & $3.405 \pm 0.089$ & 1.350 & 0.350 & $1.800 \pm 0.075$ & 2.000 & 0.750 & $6.228 \pm 0.358$ \\
\hline 1.100 & 0.650 & $4.007 \pm 0.124$ & 1.350 & 0.400 & $2.029 \pm 0.082$ & 2.000 & 0.800 & $7.471 \pm 0.483$ \\
\hline 1.100 & 0.700 & $4.723 \pm 0.140$ & 1.500 & 0.300 & $1.485 \pm 0.051$ & 2.000 & 0.850 & $8.646 \pm 0.567$ \\
\hline 1.100 & 0.750 & $5.568 \pm 0.164$ & 1.500 & 0.400 & $2.000 \pm 0.070$ & 2.000 & 0.900 & $10.142 \pm 0.723$ \\
\hline 1.100 & 0.800 & $6.591 \pm 0.232$ & 1.500 & 0.450 & $2.309 \pm 0.081$ & 2.500 & 0.300 & $1.886 \pm 0.091$ \\
\hline 1.100 & 0.850 & $7.838 \pm 0.330$ & 1.500 & 0.500 & $2.678 \pm 0.093$ & 2.500 & 0.400 & $2.397 \pm 0.134$ \\
\hline 1.100 & 0.900 & $8.973 \pm 0.375$ & 1.500 & 0.550 & $3.133 \pm 0.097$ & 2.500 & 0.450 & $2.812 \pm 0.176$ \\
\hline 1.200 & 0.500 & $2.539 \pm 0.071$ & 1.500 & 0.600 & $3.664 \pm 0.135$ & 2.500 & 0.500 & $3.186 \pm 0.203$ \\
\hline 1.200 & 0.550 & $2.984 \pm 0.085$ & 1.500 & 0.650 & $4.307 \pm 0.153$ & 2.500 & 0.550 & $3.554 \pm 0.218$ \\
\hline 1.200 & 0.600 & $3.461 \pm 0.099$ & 1.500 & 0.700 & $5.099 \pm 0.216$ & 2.500 & 0.600 & $4.249 \pm 0.276$ \\
\hline 1.200 & 0.650 & $4.093 \pm 0.141$ & 1.500 & 0.750 & $6.046 \pm 0.255$ & 2.500 & 0.650 & $4.888 \pm 0.311$ \\
\hline 1.200 & 0.700 & $4.828 \pm 0.159$ & 1.500 & 0.800 & $7.013 \pm 0.349$ & 2.500 & 0.700 & $5.791 \pm 0.417$ \\
\hline 1.200 & 0.750 & $5.749 \pm 0.191$ & 1.500 & 0.850 & $8.206 \pm 0.414$ & 2.500 & 0.750 & $6.539 \pm 0.476$ \\
\hline 1.200 & 0.800 & $6.727 \pm 0.262$ & 1.500 & 0.900 & $9.396 \pm 0.477$ & 2.500 & 0.800 & $7.733 \pm 0.659$ \\
\hline 1.200 & 0.850 & $7.896 \pm 0.363$ & 1.800 & 0.300 & $1.611 \pm 0.074$ & 2.500 & 0.850 & $8.85 \pm 0.718$ \\
\hline 1.200 & 0.900 & $9.170 \pm 0.424$ & 1.800 & 0.400 & $2.117 \pm 0.097$ & 2.500 & 0.900 & $10.366 \pm 0.924$ \\
\hline 1.300 & 0.450 & $2.227 \pm 0.068$ & 1.800 & 0.450 & $2.439 \pm 0.107$ & 4.000 & 0.300 & $2.396 \pm 0.106$ \\
\hline 1.300 & 0.500 & $2.571 \pm 0.076$ & 1.800 & 0.500 & $2.845 \pm 0.120$ & 4.000 & 0.600 & $4.929 \pm 0.385$ \\
\hline 1.300 & 0.550 & $2.993 \pm 0.086$ & 1.800 & 0.550 & $3.223 \pm 0.133$ & 4.000 & 0.900 & $11.481 \pm 1.359$ \\
\hline 1.300 & 0.600 & $3.548 \pm 0.112$ & & & & & & \\
\hline
\end{tabular}




\section{References}

[1] B.E. Poling, J.M. Prausnitz, J.P. O'Connell, The properties of Gases and Liquids, McGraw-Hill, New-York, 2001.

[2] G. Galliéro, C. Boned, A. Baylaucq, Molecular dynamics study of the LennardJones fluid viscosity: application to real fluids, Industrial Engineering and Chemical Research 44 (2005) 6963-6972.

[3] S.T. O'Connell, P.A. Thompson, Molecular dynamics-continuum hybrid computations: a tool for studying complex fluid flows, Physical Review E 52 (6) (1995) 5792-5796.

[4] R. Delgado-Buscalioni, P.V. Coveney, Continuum-particle hybrid coupling for mass, momentum, and energy transfers in unsteady fluid flow, Physical Review E 67 (046704) (2003).

[5] X.B. Nie, S.Y. Chen, W.N. E, M.O. Robbins, A continuum and molecular dynamics hybrid method for micro- and nano-fluid flow, Journal of Fluid Mechanics 500 (2004) 55-64.

[6] N.G. Hadjiconstantinou, Discussion of hybrid atomistic-continuum methods for multiscale hydrodynamics, International Journal for Multiscale Computational Engineering 2 (2) (2004) 189-202.

[7] T. Werder, H. W. Jens, P. Koumoutsakos, Hybrid atomistic-continuum method for the simulation of dense fluid flows, Journal of Computational Physics 205 (2005) 373-390.

[8] M.S. Zabaloy, V.R. Vasquez, E.A. Macedo, Viscosity of pure supercritical fluids, Journal of Supercritical Fluids 36 (2) (2005) 106-117.

[9] L.V. Woodcock, Equation of state for the viscosity of Lennard-Jones fluids, AIChE Journal 52 (2) (2006) 438-446.

[10] R. Laghaei, A. E. Nasrabad, B.C. Eu, Statistical-mechanical theory of rheology: Lennard-Jones fluids, Journal of Chemical Physics 123 (23) (2005) 1-14.

[11] M. Robles, L.I. Uruchurtu, Theoretical scheme for the shear viscosity of LennardJones fluids, Journal of Chemical Physics, 124 (9) (2006) 094112.

[12] R.J. Speedy, F.X. Prielmeier, T. Vardag, E.W. Lang, F.-D. Lüdemann, Diffusion in simple fluids, Molecular Physics 66 (1989) 577-590. 
[13] E. Ruckenstein, H. Liu, Self-Diffusion in Gases and Liquids, Industrail and Engineering Chemistry Research 36 (1997) 3927-3936.

[14] M.S. Zabaloy, V.R. Vasquez, E.A. Macedo, Description of self-diffusion coefficients of gases, liquids and fluids at high pressure based on molecular simulation data, Fluid Phase Equilibria 242 (2006) 43-56.

[15] R. Laghaei, E. Nasrabad, B. C. Eu, Excluded volume in the generic van Der Waals equation of state and the self-diffusion coefficient of the Lennard-Jones fluid, Journal of Chemical Physics 124 (2006) 154502.

[16] K. Meier, Computer simulation and interpretation of the transport coefficients of the Lennard-Jones model fluid, $\mathrm{PhD}$ thesis, University of the Federal Armed Forces, Hamburg, Germany, Shaker Publishers, Aachen, 2002.

[17] G.A. Fernandez, J. Vrabec, H. Hasse, A molecular simulation study of shear and bulk viscosity and thermal conductivity of simple real fluids, Fluid Phase Equilibria 221 (2004) 157-163.

[18] R.J. Hulse, R.L. Rowley, W.V. Wilding, Transient nonequilibrium molecular dynamic simulations of thermal conductivity: 1. Simple Fluids, International Journal of Thermophysics 26 (1) (2005).

[19] A.E Nasrabad, R. Laghaei, B.C. Eu, Molecular theory of thermal conductivity of the Lennard-Jones fluid, Journal of Chemical Physics 124 (084506) (2006).

[20] F. Müller-Plathe, A simple nonequilibrium molecular dynamics method for calculating the thermal conductivity, Journal of Chemical Physics 106 (14) (1997) 6082-6085.

[21] D.J. Evans, G. Moriss, Statistical Mechanics of Nonequilibrium Liquids, Academic Press, London, 1990, chapter 4 \& 6.

[22] B. Hafskjold, T. Ikhesoji, S.K. Ratke, On the molecular mechanism of thermal diffusion in luiquids, Mol. Phys. 80 (1993) 1389-1412.

[23] M.P. Allen, D.J. Tildesley, Computer simulation of liquids, Oxford Science publications, Oxford, 1987, chapter 2.

[24] S.R. de Groot, P. Mazur, Non-Equilibrium Thermodynamics, Dover, New York, 1984, pp. 280-281.

[25] B.D. Todd, P.J. Daivis, D. Evans, Heat flux vector in highly inhomogeneous nonequilibrium fluids, Phys. Re. E 51 (1995) 4362-4368. 
[26] G. Galliero, B. Duguay, J.-P. Caltagirone, F. Montel, Thermal diffusion sensitivity to the molecular parameters of a binary equimolar mixture, a nonequilibrium molecular dynamics approach, Fluid Phase Equilibria 208 (1-2) (2003) 171-188.

[27] H.J.C. Berendsen, J.P.M. Postma, W.F. Van Gusteren, A. Dinola, J.R. Haak, Journal of Chemical Physics 81 (1984) 3684.

[28] R.D. Mountain, System size and control parameters effects in reverse perturbation nonequilibrium molecular dynamics, Journal of Chemical Physics 124 (2006) 104109.

[29] D.M. Heyes, M.J. Cass, J.G. Powles, W.A.B. Evans, Self-Diffusion of the HardSphere Fluid: System Size Dependence and Empirical Correlations, Journal of Physical Chemistry B 111 (2007) 1455-1464.

[30] G.Galliero, Thermodiffusion dans les fluides de Lennard-Jones par dynamique moléculaire, Phd Thesis, University of Bordeaux I, France, 2003.

[31] B.A. Younglove, H.J.M. Hanley, The viscosity and thermal conductivity coefficients of gaseous and liquid argon, Journal of Physical and Chemical Reference Data 15 (4) (1986) 1323-1337

[32] E.W. Lemmon, R.T. Jacobsen, Viscosity and thermal conductivity equations for nitrogen, oxygen, argon and air, International Journal of Thermophysics 25 (1) (2004) 21-69.

[33] P.D. Neufeld, R.A. Aziz, A.R. Janzen, Empirical equations to calculate 16 of transport collision integrals-omega(L,S)' for Lennard-Jones (12-6) potential, Journal of Chemical Physics 57 (3) (1972) 1100-1102.

[34] P.M. Mathias, V.S. Parekh, E.J. Miller, Prediction and correlation of the thermal conductivity of pure fluids and mixtures, including the critical region, Industrial and Engineering Chemistry Research 41 (2002) 989-999.

[35] J. Kolafa, I. Nezbeda, The Lennard-Jones fluid: An accurate analytical and theoretically-based equation of state, Fluid Phase Equilibria 100 (1994) 1-34.

[36] J.V. Sengers, P.H. Keyes, Scaling of the thermal conductivity near the gas-liquid critical point, Physical Review Letter 26 (1971) 70-73. 
[37] D.J. Searles, D.J. Evans, H.J.M. Hanley, S. Murad, Simulations of the thermal conductivity in the vicinity of the critical point, Molecular Simulation 20 (6) (1998) 385-395.

[38] J. Eapen, J. Li, S. Yip, Mechanism of thermal transport in dilute nanocolloids, Physical review Letters 9 (2) (2007) 028302.

[39] G. Galliéro, C. Boned, A. Baylaucq, F. Montel, Molecular dynamics comparative study of Lennard-Jones $\alpha-6$ and exponential $\alpha-6$ potentials: Application to real simple fluids (viscosity and pressure), Physical Review E 73 (2006) 061201.

[40] R.A. Perkins, D.G. Friend, H.M. Roder, C.A. Nieto de Castro, Thermal conductivity surface of argon: a fresh analysis, International Journal of Thermophysics 12 (6) (1991) 965-984.

[41] D.G. Friend, J.F. Ely, H. Ingham, Thermophysical properties of methane, Journal of Physical and Chemical Reference Data 18 (2) (1989) 583-638.

[42] A. Laesecke, R. Krauss, K. Stephan, W. Wagner, Transport properties of fluid oxygen, Journal of Physical and Chemical Reference Data 19 (5) (1990) 10891122 .

[43] R.T. Jacobsen, R.B. Stewart, M. Jahangiri, Thermodynamic properties of nitrogen from the freezing line to $2000 \mathrm{~K}$ at pressures to $1000 \mathrm{MPa}$., Journal of Physical and Chemical Reference Data 15 (2) (1986) 735-909.

[44] I.G. Kaplan, Intermolecular Interactions: Physical Picture, Computational Methods and Model Potentials, John Wiley and Sons, New York, 2006.

[45] O. Suarez, I. Medina, C. Pizarro, J.L. Bueno, On predicting self-diffusion coefficients from viscosity in gases and liquids, Chemical Engineering Science 62 (2007) 6499-6515.

[46] S.A. Mikhailenko, B.G. Dudar, V.N. Derkach, V.N. Zozulya, Volume and shear viscosity of liquid argon-krypton mixtures, Soviet Journal of Low Temperature Physics 3 (6) (1977) 331-336.

[47] S.A. Mikhailenko, B.G. Dudar, V.N. Derkach, Viscoelastic properties and acoustic relaxation in liquid methane-argon mixtures, Soviet Journal of Low Temperature Physics 4 (4) (1978) 205-212. 
[48] G. Galliero, C. Boned, A. Baylaucq, F. Montel, Influence of the mass ratio on viscosity in Lennard-Jones mixtures: The one-fluid model revisited using nonequilibrium molecular dynamics, Fluid Phase Equilibria, 234 (2005) 56-63.

[49] K.C. Mo, K.E. Gubbins, Conformal solution theory for viscosity and thermal conductivity of mixtures, Molecular Physics 31 (3) (1976) 825-847.

[50] H.J.M. Hanley, D.J. Evans, Behavior of a Nonconformal mixture via computer simulation, Internantional Journal of Thermophysics 2 (1) (1981) 1-19.

[51] G. Galliero, C. Boned, A. Baylaucq, F. Montel, The van der Waals one-fluid model for viscosity in Lennard-Jones fluids: Influence of size and energy parameters, Fluid Phase Equilibria 245 (2006) 20-25. 


\section{Figures Captions:}

Figure 1: A sketch of the simulation box subdivided into $\mathrm{N}_{\mathrm{s}}$ slabs. The dark frame indicates the simulation cell. Slabs 1 and $\mathrm{N}_{\mathrm{s}}$ are the "hot" ones. Slabs $\mathrm{N}_{\mathrm{s}} / 2$ and $\mathrm{N}_{\mathrm{s}} / 2+1$ (black layers) are the cool ones. Kinetic energy is artificially transferred from the cool to the hot slabs and then flows back by thermal conduction. The temperature profile is calculated by determining the temperatures in the intervening slabs ( 3 to $\mathrm{N}_{\mathrm{s}} / 2-2$ and $\mathrm{N}_{\mathrm{s}} / 2+3$ to $\mathrm{N}_{\mathrm{s}}-2$ ).

Figure 2: Typical temperature profile in the simulation box, subdivided into 32 slabs, for $\mathrm{T}^{*}=1, \rho^{*}=0.8, \mathrm{~N}=1500$ atoms, shown as a function of slab number. The symbols represent the layer average temperature (errors bars are smaller than the size of the symbols and have been omitted).

Figure 3: Evolution of the reduced thermal conductivity as a function of $N^{-1 / 2}$, for different thermodynamic states: $\circ, \rho^{*}=0.3$ and $T^{*}=1.5 ; \nabla, \rho^{*}=0.3$ and $T^{*}=2.5 ; \square, \rho^{*}=0.8$ and $T^{*}=1 ; \diamond, \rho^{*}=0.8$ and $T^{*}=2.5$. Dashed lines correspond to the linear regression.

Figure 4: Dependence of the three contributions of thermal conductivity versus the size of the system for two different thermodynamic states, $T^{*}=1, \rho^{*}=0.8$, grey symbols, $T^{*}=2.5, \rho^{*}=0.3$, open symbols. Circles correspond to $\lambda_{k}$, down triangles to $\lambda_{p}$ and squares to $\lambda_{c o}$.

Figure 5: Evolution of the reduced thermal conductivity as a function of the cutoff radius $r_{c}$, for different thermodynamic states: $\circ, \rho^{*}=0.3$ and $T^{*}=1.5 ; \nabla, \rho^{*}=0.7$ and $T^{*}=1 ; \square, \rho^{*}=0.8$ and $T^{*}=1 ; \diamond, \rho^{*}=0.9$ and $T^{*}=1$.

Figure 6: Critical contribution to the thermal conductivity along the isotherm $T^{*}=1.35$. Circles correspond to our simulations, down triangles to those of Meier [11] and the dashed line to the results provided by the correlation, eq. (28).

Figure 7: Deviations between MD results and those given by the correlation, eq. (23), versus $T^{*}$, left and $\rho^{*}$, right. Circles correspond to our simulations, squares to those of Hulse et al. [13], down triangles to those of Nasrabad et al. [14] and diamonds to those of Mountain [28]. 
Figure 8: Contributions (in \%) to the heat flux of the three terms, $\mathrm{J}_{\mathrm{k}}$, circles, $\mathrm{J}_{\mathrm{p}}$, down triangles, $\mathrm{J}_{\mathrm{co}}$, squares along three isotherms, $\mathrm{T}^{*}=1$ open symbols, $\mathrm{T}^{*}=1.35$ dark symbols and $\mathrm{T}^{*}=2.5$ grey symbols.

Figure 9: Thermal conductivity deviations given by the correlation, eq. (23), on Argon [40], filled circles, Methane [41], open down triangles, Oxygen [42], filled squares and Nitrogen [43], open diamonds.

Figure 10: Thermal conductivity deviations for the system Ar-Kr, open symbols, and Ar- $\mathrm{CH}_{4}$, full symbols, given by the correlation, eq. (23), combined with the vdW1 and LB combining rules, relatively to the values of Mikhailenko et al. [46-47] : squares, $T=100 \mathrm{~K}$, up triangles, $T=110 \mathrm{~K}$, down triangles, $T=120 \mathrm{~K}$, circles, $T=130 \mathrm{~K}$ and diamonds, $T=140 \mathrm{~K}$. 


\section{Tables:}

Table 1: Deviations between our results on thermal conductivity (including finite size corrections eqs. (18-19)) and those coming from references [14,28].

\begin{tabular}{|l|l|l|l|}
\hline & AAD (\%) & $\Delta_{\max }(\%)$ & Bias (\%) \\
\hline Nasrabad et al. [14] (64 points) & 3.59 & 8.28 & -3.42 \\
\hline Mountain [28] (NEMD, 14 points) & 3.62 & 8.29 & -3.18 \\
\hline Mountain [28] (EMD, 14points) & 4.57 & 16.79 & -3.27 \\
\hline
\end{tabular}

Table 2: Coefficients used in the thermal conductivity correlation (eqs.(26), (27), (28)).

\begin{tabular}{|l|l|l|l|l|l|}
\hline C & D & E & F & a & b \\
\hline 0.00801212 & 0.09769765 & 0.00566383 & 4.69930247 & 0.11 & 0.45 \\
\hline
\end{tabular}

Table 3: Molecular parameters of the studied compounds [39].

\begin{tabular}{|l|l|l|}
\hline Compound & $\sigma(\AA)$ & $\varepsilon(\mathrm{J} / \mathrm{mol})$ \\
\hline Argon & 3.408 & 989 \\
Krypton & 3.645 & 1372.8 \\
Methane & 3.704 & 1212.6 \\
Oxygen & 3.368 & 994.1 \\
Nitrogen & 3.614 & 820.5 \\
\hline
\end{tabular}

Table 4: Results obtained for different pure compounds on a wide variety of thermodynamic states.

\begin{tabular}{|l|l|l|l|l|l|}
\hline Compound & $\begin{array}{l}\rho_{\min }-\rho_{\max } \\
\left(\mathrm{Kg} / \mathrm{m}^{3}\right)\end{array}$ & $\begin{array}{l}\mathrm{T}_{\min }-\mathrm{T}_{\max } \\
(\mathrm{K})\end{array}$ & $\mathrm{AAD}(\%)$ & $\Delta \max (\%)$ & database \\
\hline Argon & $3-1410$ & $90-452$ & 5.1 & 13.6 & Perkins et al. [40] \\
Methane & $1-446$ & $114-572$ & 21.0 & 54.7 & Friend et al. [41] \\
Oxygen & $3-1238$ & $93-464$ & 14.1 & 27.0 & Laesecke et al. [42] \\
Nitrogen & $2-867$ & $76-379$ & 16.0 & 21.6 & Jacobsen et al. [43] \\
\hline
\end{tabular}


Table 5: Results obtained for simple binary mixtures using the scheme proposed in this work (with LB combining rules and vdW approximation).

\begin{tabular}{|l|l|l|l|l|l|}
\hline Mixture & $\rho_{\min }-\rho_{\max }\left(\mathrm{Kg} / \mathrm{m}^{3}\right)$ & $\mathrm{T}_{\min }-\mathrm{T}_{\max }(\mathrm{K})$ & $\mathrm{AAD}(\%)$ & $\Delta \max (\%)$ & Database \\
\hline $\mathrm{Ar}+\mathrm{Kr}$ & $942-2412$ & $120-140$ & 9.3 & 22.4 & Mikhailenko \\
$\mathrm{Ar}+\mathrm{CH}_{4}$ & $380-1312$ & $100-140$ & 3.3 & 11 & et al. [46-47] \\
\hline
\end{tabular}


Figures:

Figure 1

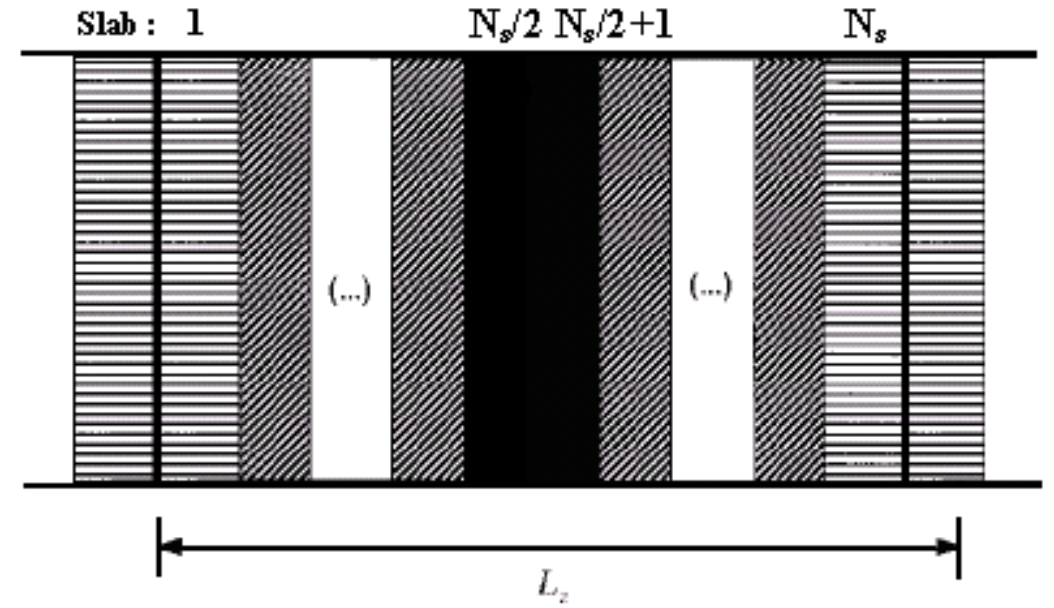

Figure 2

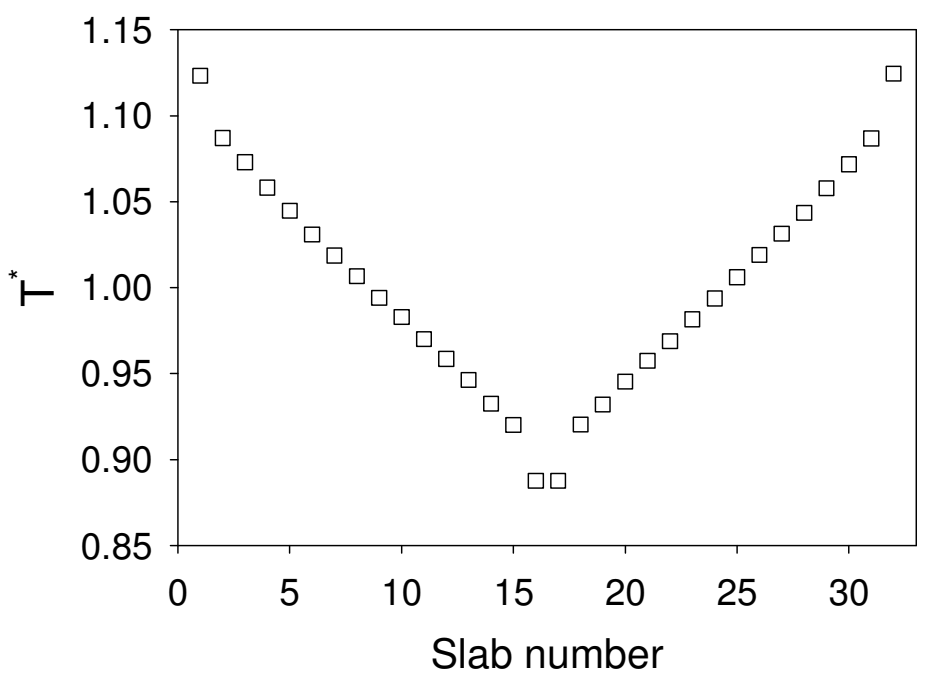


Figure 3

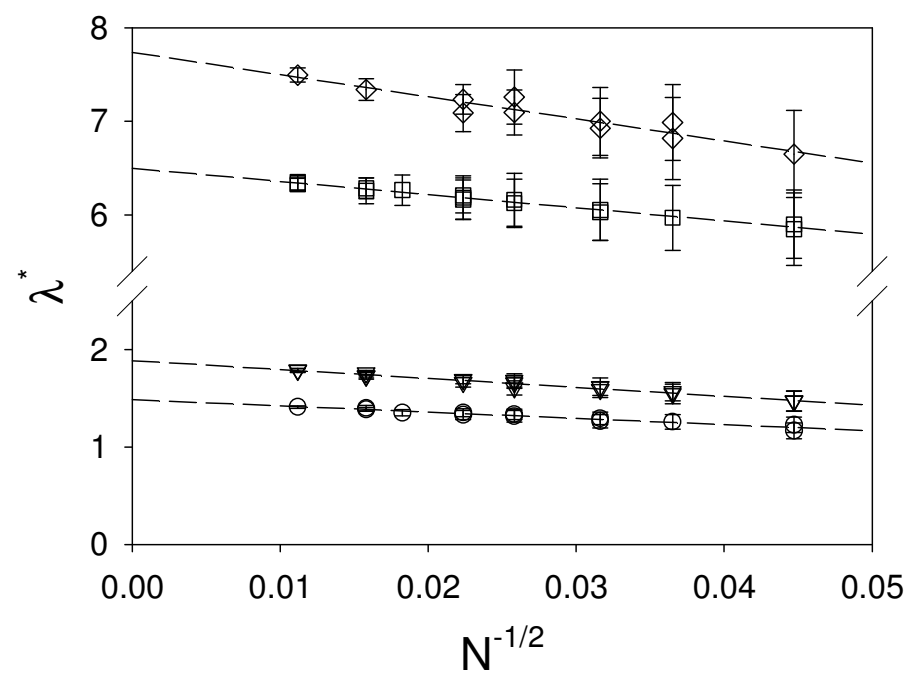

Figure 4

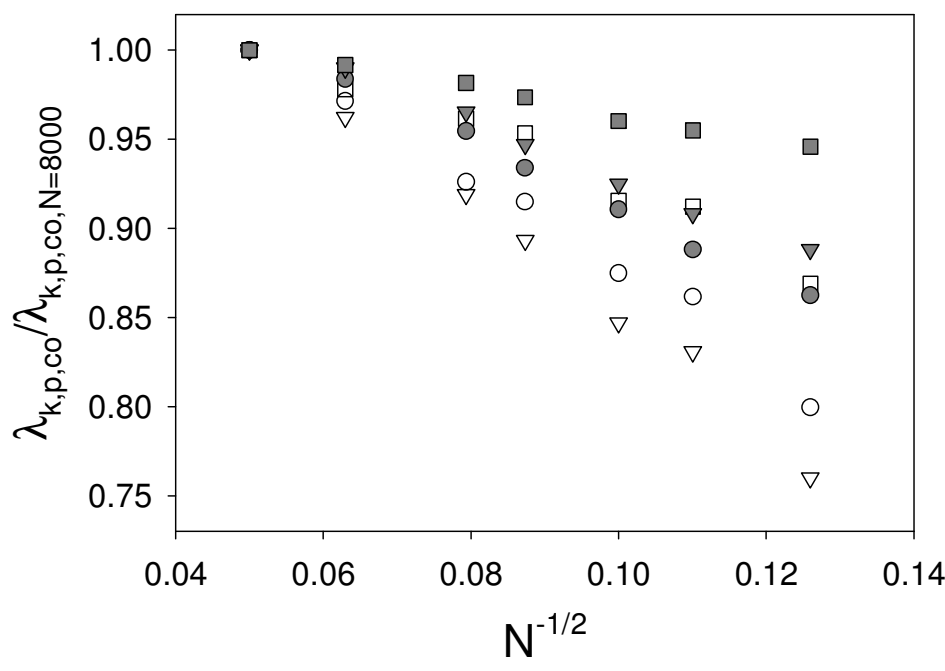


Figure 5

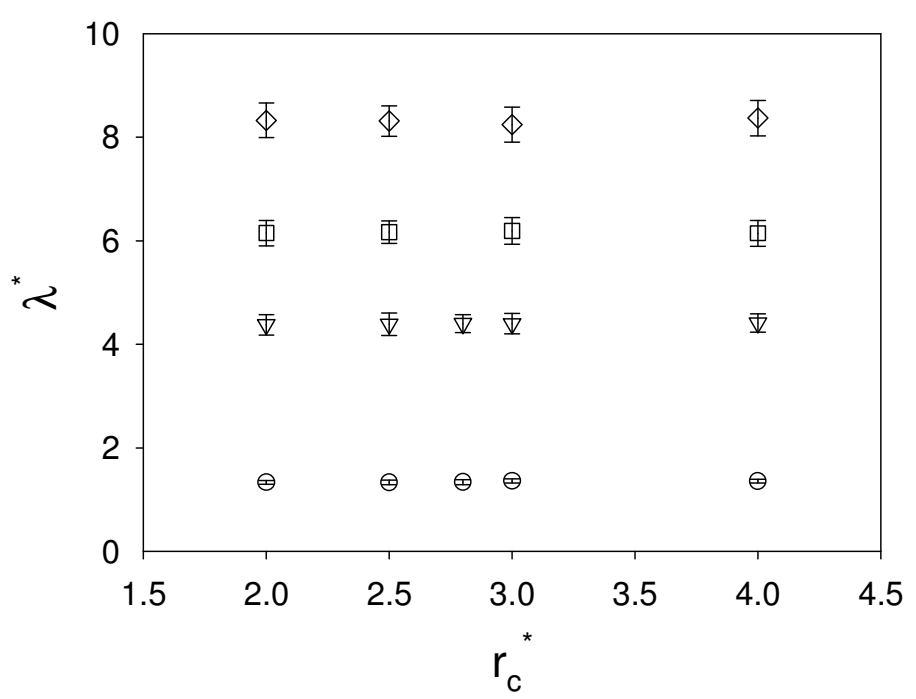

Figure 6

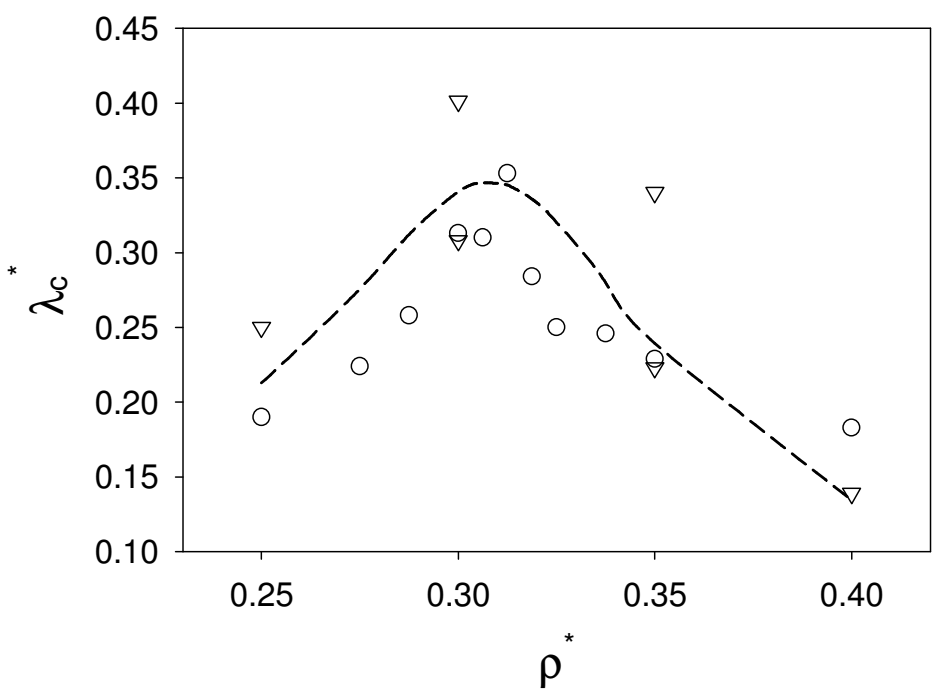


Figure 7
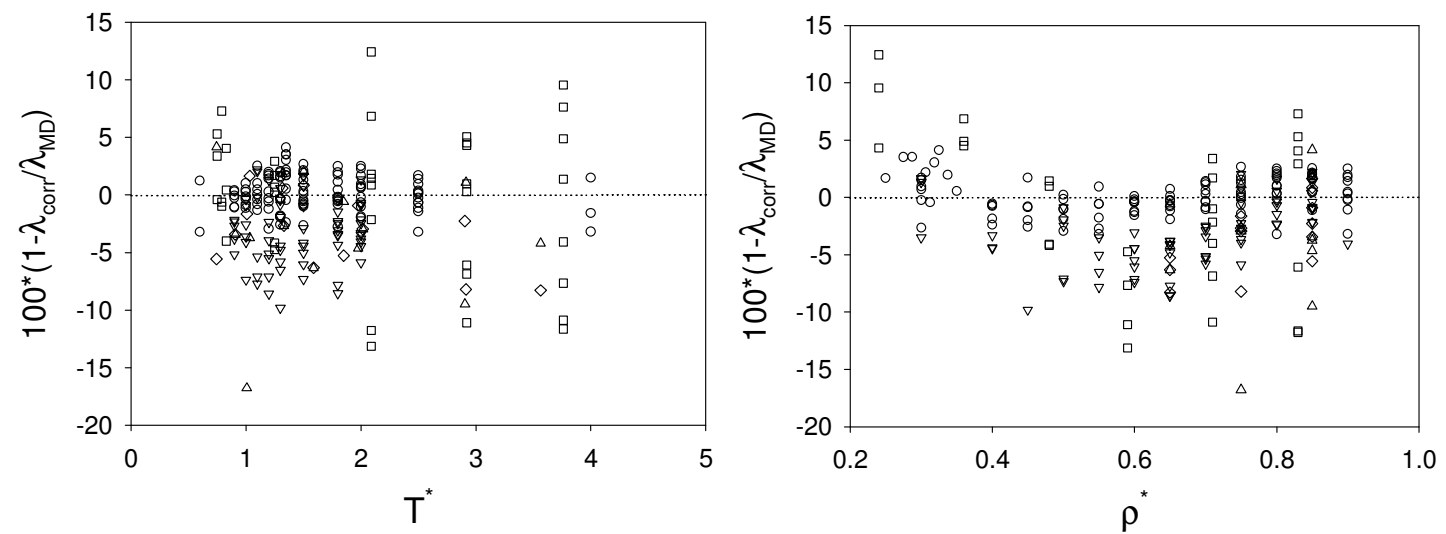

Figure 8

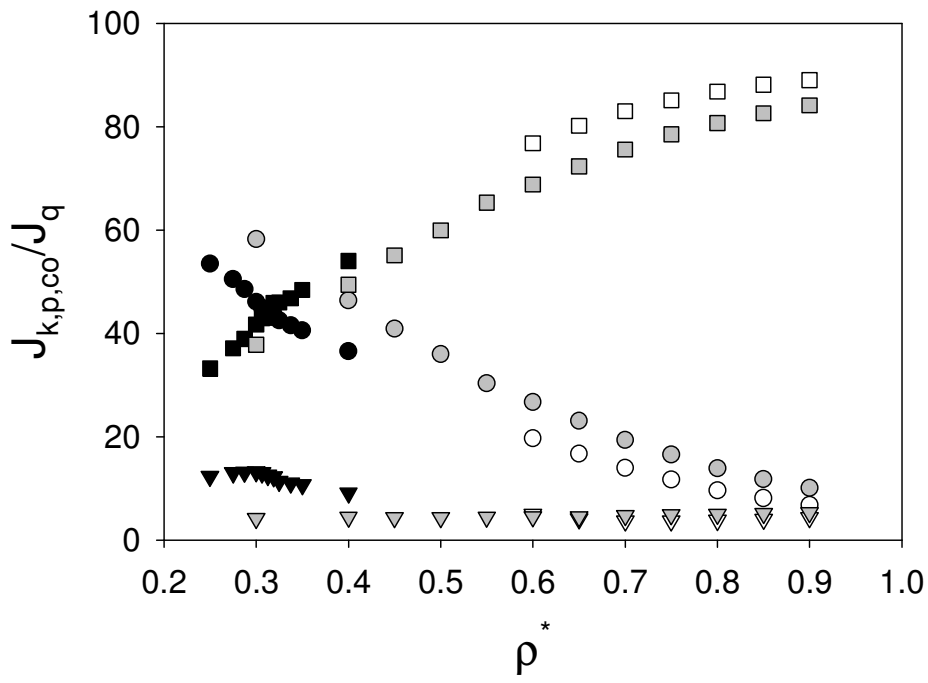


Figure 9

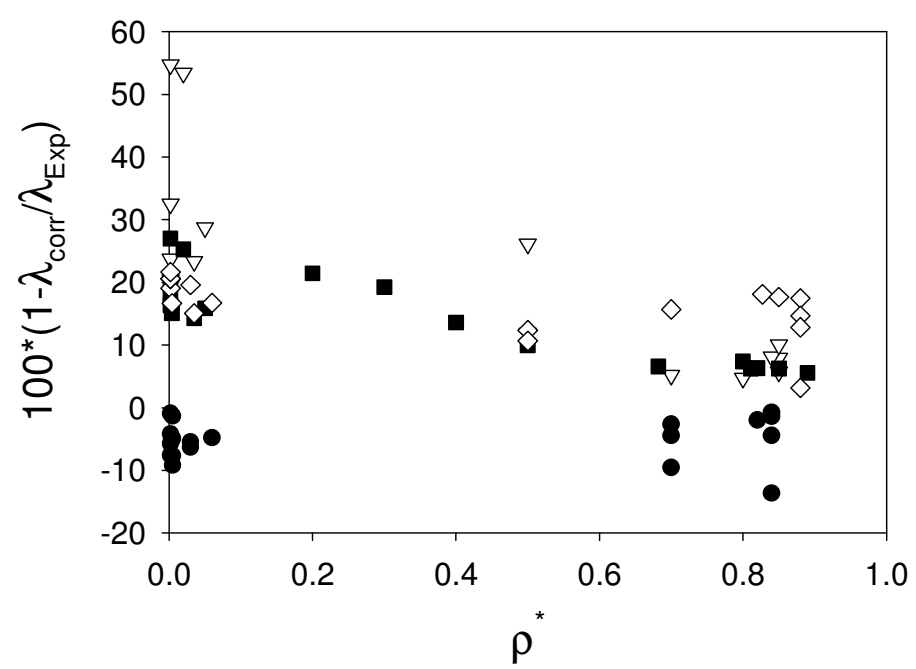

Figure 10

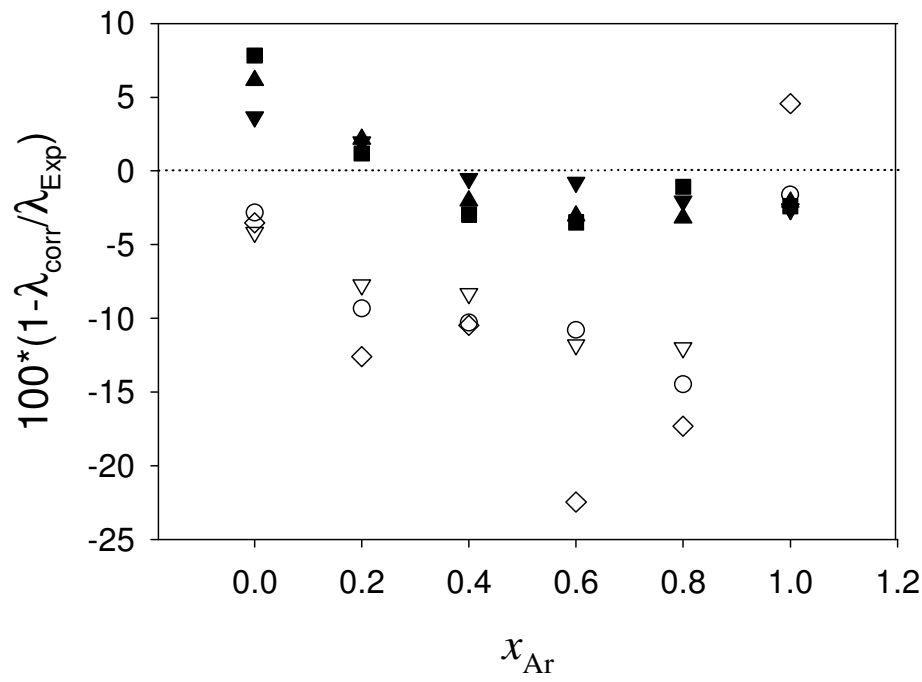

